\title{
Perturbative SO(10) GUT and the Minimal Higgs Sector
}

\author{
Sören Wiesenfeldt and Scott Willenbrock \\ Department of Physics, University of Illinois at Urbana-Champaign, \\ 1110 West Green Street, Urbana, IL 61801, USA
}

\begin{abstract}
The breaking of $\mathrm{SO}(10)$ to $\mathrm{SU}(3)_{C} \times \mathrm{U}(1)_{\mathrm{EM}}$ can be accomplished by just four Higgs fields: the symmetric rank-two tensor, $S(54)$; a pair of spinors, $C(16)$ and $\bar{C}(\overline{16})$; and a vector, $T(10)$. This setup is also able to generate realistic fermion masses. The heavy color triplets in the vector and spinor fields mediate proton decay via dimension-five operators. The experimental bounds on proton decay constrain the structure and size of the Yukawa operators.
\end{abstract}

$\mathrm{SO}(10)$ [1] is arguably the most natural grand-unified theory (GUT): both the standard model (SM) gauge and matter fields are unified, introducing only one additional matter particle, the right-handed neutrino. It is an anomaly-free theory and therefore explains the intricate cancellation of the anomalies in the standard model [2]. Moreover, it contains $B-L$ as a local symmetry, where $B$ and $L$ are baryon and lepton number, respectively; the breaking of $B-L$ naturally provides light neutrino masses via the seesaw mechanism.

Despite these attractive features, the breaking of the GUT symmetry has remained a problem in model building. Generally, two avenues have been pursued. One makes use of large representations, $\Phi(210)$, $\Sigma(126), \bar{\Sigma}(\overline{126})$, and $T(10)$ [3]. This approach has the advantage of a fully renormalizable superpotential and automatic $R$ parity. The unified gauge coupling, however, diverges just above the GUT scale, indicating that new physics must enter. Since this new physics is close to the GUT scale, it potentially has a large effect on the predictions of the model. In addition, this scenario is insufficient to reproduce the fermion mass spectrum, have successful gauge unification, and fulfill the proton decay constraints at the same time [4. Hence, a realistic renormalizable model requires at least another Higgs field [5].

Models with only small representations remain perturbative up to the Planck scale [6] and also have the potential to arise from string theory. They introduce a moderate number of new fields, yielding only small threshold corrections at $M_{\mathrm{GUT}}$. In the supersymmetric version of this scenario, higher-dimensional operators, suppressed by powers of a more fundamental scale $M$ (such as the Planck scale, $M_{\mathrm{P}}=\left(8 \pi G_{N}\right)^{-1 / 2}=$ $2 \cdot 10^{18} \mathrm{GeV}$, or the string scale in the weakly coupled heterotic string, $M_{\mathrm{S}} \approx 5 \cdot 10^{17} \mathrm{GeV}$ ), are essential to achieve the breaking to the $\mathrm{SM}$ group, $\mathrm{G}_{\mathrm{SM}}=\mathrm{SU}(3)_{C} \times \mathrm{SU}(2)_{L} \times \mathrm{U}(1)_{Y}$ [7]. Moreover, these operators play an important role in fermion masses and mixings [8, 9]. First, they generate Majorana masses for the right-handed neutrinos in the desired range, $M_{\mathrm{GUT}}^{2} / M \sim 10^{14} \mathrm{GeV}$. Thus neutrino masses require the vacuum expectation value (vev) of the $B-L$ breaking field to be of the order of $M_{\mathrm{GUT}}$. Second, they naturally explain why certain relations, such as the bottom-tau unification, are only approximately realized. Finally, they alter the couplings of the matter fields to the Higgs color triplets relative to the weak doublets, such that the proton decay rate via dimension-five operators can be significantly reduced [10].

Models with small representations usually use the antisymmetric second-rank tensor, $A(45)$, together with a pair of spinors, $C(16)$ and $\bar{C}(\overline{16})$. In order to give GUT-scale masses to the color-triplet Higgs fields, one must introduce two ten-dimensional Higgs fields because the term $T A T$ vanishes due to the antisymmetry of $A$. This setup can implement the Dimopoulos-Wilczek mechanism [11, 12], yielding automatic mass splitting of the (light) Higgs doublets and (heavy) color triplets. However, the realization of this mechanism requires a second pair of spinorial Higgs fields and an extensive set of global symmetries [7]. Thus it is important to investigate alternative scenarios.

\footnotetext{
${ }^{1}$ One attempt has been to realize the GUT gauge symmetry in more than four space-time dimension and use GUT-symmetry breaking boundary conditions on an orbifold for the breaking to the SM. This scenario yields doublet-triplet splitting and avoids the dangerous dimension-five proton-decay operators [13.
} 
Although the breaking of $\mathrm{SO}(10)$ to the standard model by the symmetric rank-two tensor, $S(54)$, and a spinorial, $B-L$ breaking field is a standard textbook example [14], a realistic supersymmetric model has never been worked out.2 The reason might be twofold. First, this scenario does not allow for the Dimopoulos-Wilczek mechanism, so we have to accept fine-tuning to have one pair of Higgs doublets light. Second, $S(54)$ breaks $\mathrm{SO}(10)$ to the Pati-Salam group, $\mathrm{G}_{\mathrm{PS}}=\mathrm{SU}(4)_{C} \times \mathrm{SU}(2)_{L} \times \mathrm{SU}(2)_{R}$. Hence, in contrast to $A$, it does not break $\mathrm{SU}(4)_{C}$ and therefore preserves the unification of down-quark and charged-lepton masses. Thus, with only small Higgs representations, it is more involved to generate realistic quark and lepton masses.

The use of $S(54)$, however, has advantages over both models described above. In contrast to the model with large representations, the gauge coupling remains perturbative up to $M_{\mathrm{P}}$ and unlike the scenario with $A(45)$, it requires only one ten-dimensional Higgs field for the electroweak symmetry breaking. 35 In this letter we demonstrate that $\mathrm{SO}(10)$ can be broken to $\mathrm{SU}(3)_{C} \times \mathrm{U}(1)_{\mathrm{EM}}$ by a set of four Higgs fields: $S(54), C(16)$, $\bar{C}(\overline{16})$, and $T(10)$. Analyzing the higher-dimensional operators, we show that realistic fermion masses and mixings can be generated and a sufficiently low proton decay rate can be obtained.

Breaking of SO(10). The $\mathrm{SO}(10)$ symmetry is broken by the vevs of $S(54), C(16)$, and $\bar{C}(\overline{16})$ [15]. The superpotential can be split into three parts,

$$
W=W_{S}+W_{C}+W_{S C} .
$$

The first part yields the breaking to $\mathrm{SO}(6) \times \mathrm{SO}(4) \simeq \mathrm{G}_{\mathrm{PS}}$,

$$
W_{S}=\frac{1}{2} M_{S} \operatorname{tr} S^{2}+\frac{1}{3} \lambda_{S} \operatorname{tr} S^{3}, \quad\langle S\rangle=v_{s} \operatorname{diag}(2,2,2,2,2,2 ;-3,-3,-3,-3), \quad v_{s}=\frac{M_{S}}{\lambda_{S}} .
$$

$\langle S\rangle$ is chosen such that the first six entries preserve $\mathrm{SO}(6) \simeq \mathrm{SU}(4)_{C}$, whereas the last preserve $\mathrm{SO}(4) \simeq$ $\mathrm{SU}(2)_{L} \times \mathrm{SU}(2)_{R}$. The second part of the superpotential describes the breaking of $\mathrm{SO}(10)$ to $\mathrm{SU}(5)$ by the vevs of the spinorial representations. It requires the inclusion of one dimension-five operator, f $^{2}$

$$
W_{C}=M_{C} C \bar{C}+\frac{\lambda_{C}}{2 M}(C \bar{C})^{2}, \quad\langle C \bar{C}\rangle=-\frac{M_{C} M}{\lambda_{C}} \equiv v_{c}^{2} .
$$

Together, these vevs break $\mathrm{SO}(10)$ to the intersection of $\mathrm{SU}(5)$ and $\mathrm{G}_{\mathrm{PS}}$, namely the standard model group G SM

At the renormalizable level, the Higgs fields do not couple in the potential. Therefore we have two independent global SO(10) symmetries and the total number of Goldstones is $(45-24)+(45-21)=45$. Only $45-12=33$ true Goldstones are eaten by gauge bosons, so there are 12 pseudo-Goldstones. These are a vectorial pair of quark-doublet fields, $Q\left(3,2, \frac{1}{6}\right)+\bar{Q}\left(\overline{3}, 2,-\frac{1}{6}\right)$, contained both in $C+\bar{C}$ and in $S$. This extra global symmetry, however, is accidental, and is violated by the non-renormalizable interaction term

\footnotetext{
${ }^{2}$ The Higgs sector for this setup was considered for the non-supersymmetric case 15 and also for the supersymmetric scenario, but with two ten-dimensional fields $[16$. In several supersymmetric models, $S$ is used as an additional field to achieve the symmetry breaking [17].

${ }^{3}$ Recently, it was shown that $\mathrm{SO}(10)$ can be broken to $\mathrm{G}_{\mathrm{SM}}$ by a single pair of vector-spinors, $\Upsilon_{(+)}(144)+\Upsilon_{(-)}(\overline{144})[18]$; however, in order to generate realistic fermion masses, one needs to introduce additional matter fields, such as $10_{M}$ and $45_{M}$ 19]. We will not consider this approach in this letter.

${ }^{4}$ Alternatively, we may introduce a singlet $X$ such that

$$
W_{C}=X\left(\lambda_{C} C \bar{C}-M_{C}^{2}\right), \quad \frac{\partial W_{C}}{\partial X}=\lambda_{C} C \bar{C}-M_{C}^{2} \Rightarrow\langle C \bar{C}\rangle=\frac{M_{C}^{2}}{\lambda_{C}}, \quad \frac{\partial W_{C}}{\partial C}=\lambda_{C} X \bar{C} \Rightarrow\langle X\rangle=0,
$$

avoiding the dimension-five operator. The price we pay is to introduce a singlet which in general can couple to the other fields as well.
} 
between the different Higgs fields,

$$
W_{S C}=\frac{\xi_{C}}{2 M} C \bar{C} S^{2}
$$

The pseudo-Goldstones acquire masses of order $v_{s} v_{c} / M$. Their presence changes the 1-loop coefficients of the $\beta$ function of the gauge couplings $\left(\alpha_{1}, \alpha_{2}, \alpha_{3}\right)$ by $\Delta b=\left(\frac{1}{5}, 3,2\right)$ such that $b=\left(\frac{34}{5}, 4,-1\right)$ [20]. Hence, they mostly modify the running of $\alpha_{2}$ and $\alpha_{3}$. Fig. 1 1 shows the impact of the pseudoGoldstones, at a mass of $3 \cdot 10^{14} \mathrm{GeV}$, on the running of the gauge couplings in the MSSM at one loop. Since the gauge coupling unification is upset, it is clear that the pseudoGoldstone masses must be close to the GUT scale in order to preserve gauge coupling unification. This can be achieved provided that $v_{c}>v_{s}$; if $\lambda_{C} \sim \frac{M_{C}}{M}$, the pseudo-Goldstones become as heavy as $v_{s}$. In this scenario, $\mathrm{SO}(10)$ is first broken to $\mathrm{SU}(5)$ at $v_{c}$, and then to the $\mathrm{SM}$ at $v_{s}$. A drawback to this scenario is that $v_{c} \sim M$, so even higher-dimensional operators obtained by adding powers of $(C \bar{C}) / M^{2},(C / M)^{4}$, and $(\bar{C} / M)^{4}$ are not necessarily suppressed.

The renormalizable part of the superpotential for the electroweak symmetry breaking reads
Figure 1: The running of the gauge couplings at 1-loop in the MSSM (dashed) and with the inclusion of the pseudo-Goldstones at $3 \cdot 10^{14} \mathrm{GeV}$ (solid). The MSSM couplings unify at around $2 \cdot 10^{16} \mathrm{GeV}$, while the inclusion of the pseudo-Goldstones at a lower scale disrupts the unification.

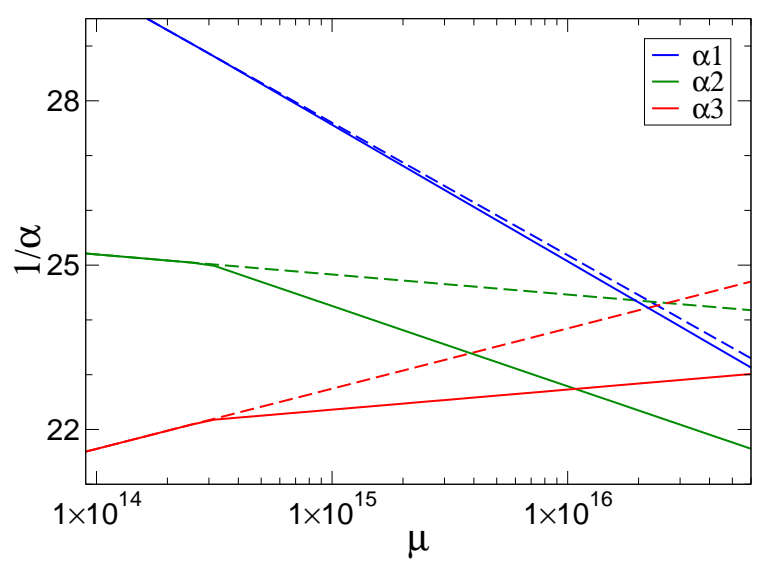

$$
W_{T}=\frac{1}{2} M_{T} T^{2}+\frac{1}{2} \lambda_{T} T S T+\xi_{T} C C T+\xi_{T}^{\prime} \bar{C} \bar{C} T .
$$

The mass matrix for the weak doublets, $H_{u}(T), H_{d}(T), \widetilde{H}_{u}(\bar{C})$, and $\widetilde{H}_{d}(C)$, is such that without fine-tuning, all doublets have GUT-scale masses,

$$
\left(\begin{array}{cc}
H_{u} & \widetilde{H}_{u}
\end{array}\right)\left(\begin{array}{cc}
M_{T}-3 \lambda_{T} v_{s} & \xi_{T} v_{c} \\
\xi_{T}^{\prime} v_{c} & M_{C}+\frac{\lambda_{C}}{M} v_{c}
\end{array}\right)\left(\begin{array}{c}
H_{d} \\
\widetilde{H}_{d}
\end{array}\right) .
$$

This is the well-known doublet-triplet splitting problem. In order to arrange for two light doublets, we have to impose that the determinant of this mass matrix vanishes, up to weak-scale terms. As a result, the two light doublets are combinations of the four doublets in $T, C$, and $\bar{C}$, and all doublets acquire weak-scale vevs. 1 This is crucial in order to derive a realistic fermion mass spectrum, as we will discuss in the next section [8, 9]. We will denote the $\mathrm{SU}(5)$-singlet and $\mathrm{SU}(2)$-doublet vevs of $C$ as $\left\langle C^{\mathrm{GUT}}\right\rangle$ and $\left\langle C^{\mathrm{EW}}\right\rangle$ and similarly the vevs of $\bar{C}$.

For later purposes, note that due to the vev of $S$ in Eq. (21), the color triplets of any ten-dimensional representation occupy the first six entries, whereas the last four are for the weak doublets. For $T$, e.g., we may write $T=\left(H_{C}, \bar{H}_{C} ; H_{u}, H_{d}\right)$, where $H_{C}$ and $\bar{H}_{C}$ are the proton-decay-mediating color triplets.

Fermion Masses. There is only one renormalizable operator that generates masses for the matter fields $16_{i}, i=1,2,3$,

$$
W_{Y}^{(4)}=h_{T}^{i j} 16_{i} 16_{j} T
$$

\footnotetext{
${ }^{5}$ This is similar to the scenario with large representations, where the doublets mix through the vev of $\Phi(210)$ and the light doublets are mixtures of those in $T(10), \Sigma(126), \Sigma(\overline{126})$, and $\Phi(210)$. 3 .
} 
this term predicts Yukawa unification at $M_{\mathrm{GUT}}, h_{u}=h_{d}=h_{e}=h_{\nu}^{\mathrm{D}}$. To have a realistic mass pattern, we need to consider higher-dimensional operators. At dimension five, we have

$$
W_{Y}^{(5)}=\frac{1}{M}\left[h_{S}^{i j} 16_{i} 16_{j} T S+h_{C}^{i j} 16_{i} 16_{j} C C+h_{\bar{C}}^{i j} 16_{i} 16_{j} \bar{C} \bar{C}\right] .
$$

These operators can be generated by integrating out heavy fields in various $\mathrm{SO}(10)$ representations, as indicated in Table 1 .

The first term in Eq. (8) contributes equally to the mass matrices of quarks and leptons since the vev of $S$ conserves $\mathrm{SU}(4)_{C}$. This is different from the scenario with the antisymmetric representation, which breaks $\mathrm{SO}(10)$ to the left-right symmetric group, $\mathrm{SU}(3)_{C} \times \mathrm{SU}(2)_{L} \times \mathrm{SU}(2)_{R} \times \mathrm{U}(1)_{B-L}$. The second term contributes (in equal measure) to the masses of down quarks and charged leptons, whereas the last term generates Dirac masses for up quarks and neutrinos as well as Majorana masses for the right-handed neutrinos [21]. These terms allow for a non-trivial CKM-matrix and degrade the Yukawa relations to $h_{d}=h_{e}$. The relation $h_{u}=h_{\nu}^{\mathrm{D}}$ is violated by the operators $\left[16_{i} \bar{C}\right]\left[16_{j} \bar{C}\right]$, obtained by integrating out heavy fields in either the singlet or adjoint representation, as shown in Table 1 [22].

In order to alter the unification of down quark and charged lepton masses, we have to go further and consider terms containing both $S$ and $C$ fields. The lowest operator is of dimension six,

$$
W_{Y}^{(6)}=\frac{h_{S C}^{i j}}{M^{2}} 16_{i} 16_{j} C C S
$$

suppressed by $v_{c} v_{s} / M^{2}$. For $M=M_{\mathrm{P}}$, this suppression factor is of similar order of magnitude as the ratio of the strange quark mass and the weak scale, namely $10^{-3}$. Thus this term is large enough to account for the difference of strange-quark and muon masses. We will now show that this term does indeed violate the equality of down-quark and charged-lepton masses.

Actually, there are two distinct operators in Eq. (9). To see that, let us recall that the Higgs field 120 contains two pairs of weak doublets. The first pair is that of the $\mathrm{SU}(5)$-fields $5+\overline{5}$ of $\mathrm{SU}(5)$ and $(1,2,2)$ of $\mathrm{G}_{\mathrm{PS}}$, and it couples equally to down quarks and charged fermions. (The same applies to the pair of Higgs doublets in T.) The second pair of doublets is contained in $45+\overline{45}$ of $\mathrm{SU}(5)$ and $(15,2,2)$ of $\mathrm{G}_{\mathrm{PS}}$. Here the couplings to the fermion fields pick up a $B-L$ factor, modifying the Yukawa unification [23].

In Eq. (9), the fermions effectively couple to 10,120 , or $\overline{126}$ fields in the $16 \times 16 \times 54$ decomposition. We expect two types of couplings to be present, one that yields Yukawa unification, and one that violates it. Let us demonstrate that the operator in Eq. (9) can indeed modify the Yukawa unification by integrating out heavy $10_{M}=\left(D, D^{c} ; L, L^{c}\right)_{M}$ fields. In this case, the two types of couplings are $h_{S C}^{(A) i j}\left[16_{i} 16_{j}\right]_{10} S[C C]_{10}$ and $h_{S C}^{(B) i j}\left[16_{i} C\right]_{10} S\left[16_{j} C\right]_{10}$. The first operator gives equal contributions to down quark and charged lepton masses, so let us study the second in detail.

The coupling of the matter fields $16_{i}$ and $10_{M}$ to $C^{\mathrm{EW}}$ (where $\widetilde{H}_{d}(C)$ acquires its vev) yields

$$
16_{i} 10_{M} C^{\mathrm{EW}} \ni\left(d_{i} D_{M}^{c}+e_{i}^{c} L_{M}+\nu_{i}^{c} L_{M}^{c}\right) \widetilde{H}_{d},
$$

in terms of $\mathrm{SO}(10)$ and $\mathrm{SM}$ fields. The coupling to $C^{\mathrm{GUT}}$ (where the $\mathrm{SM}$ singlet component, $N(C)$ ), acquires its vev) gives

$$
16_{j} 10_{M} C^{\mathrm{GUT}} \ni\left(d_{j}^{c} D_{M}+L_{j} L_{M}^{c}\right) N .
$$

As noted above, the color triplets of the $\mathrm{SO}(10)$ vector live in the first six entries, the doublets in the last four. Then we may write $\left[16\left\langle C^{\mathrm{EW}}\right\rangle\right]=\left(d ; e^{c}, 0\right) v_{d}$ and $\left[16\left\langle C^{\mathrm{GUT}}\right\rangle\right]=\left(d^{c} ; L\right) v_{c}$, where $\left\langle\widetilde{H}_{d}\right\rangle=v_{d}$. Now it is straightforward to see that this operator indeed violates the Yukawa unification,

$$
\frac{h_{S C}^{(B) i j}}{M^{2}}\left[\left(d ; e^{c}, 0\right)_{i}\left\langle C^{\mathrm{EW}}\right\rangle\right]\langle S\rangle\left[\left(d^{c} ; L\right)_{j}\left\langle C^{\mathrm{GUT}}\right\rangle\right]=h_{S C}^{(B) i j}\left(2 d_{i} d_{j}^{c}-3 e_{i}^{c} e_{j}\right) v_{d} \frac{v_{c} v_{s}}{M^{2}}
$$


Table 1: Yukawa operators up to dimension six and their relative contributions to the fermion mass matrices (left columns) and baryon and lepton number violating couplings (right columns). For various operators, there exist several options to contract the indices. For $16_{i} 16_{j} \bar{C} \bar{C}$, these lead to different results as indicated by the heavy field in brackets integrated out to generate the operator. The label (A) indicates the grouping $\left[16_{i} 16_{j}\right] S[C C]$, while $(B)$ indicates $\left[16_{i} C\right] S\left[16_{j} C\right]$.

\begin{tabular}{|c|c|c|c|c|c|c|c|c|c|c|}
\hline operator & & & $Y_{u}$ & $Y_{d}$ & $Y_{e}$ & $Y_{\nu}^{\mathrm{D}}$ & $Y_{q q}$ & $Y_{q l}$ & $Y_{u d}$ & $Y_{u e}$ \\
\hline$h_{T}^{i j} 16_{i} 16_{j} T$ & & & 1 & 1 & 1 & 1 & 1 & 1 & 1 & 1 \\
\hline$h_{S}^{i j} 16_{i} 16_{j} T S$ & & & -3 & -3 & -3 & -3 & 2 & 2 & 2 & 2 \\
\hline$h_{C}^{i j} 16_{i} 16_{j} C C$ & & & - & 1 & 1 & - & - & 1 & 1 & - \\
\hline \multirow[t]{3}{*}{$h_{\bar{C}}^{i j} 16_{i} 16_{j} \bar{C} \bar{C}$} & & {$[1]$} & - & - & - & 1 & - & - & - & - \\
\hline & & {$[10]$} & 1 & - & - & 1 & 1 & - & - & 1 \\
\hline & & {$[45]$} & 8 & - & - & 3 & 8 & - & - & 8 \\
\hline$h_{S S}^{i j} 16_{i} 16_{j} T S S$ & & & 9 & 9 & 9 & 9 & 4 & 4 & 4 & 4 \\
\hline \multirow[t]{2}{*}{$h_{S C}^{i j} 16_{i} 16_{j} C C S$} & $(A)$ & & - & -3 & -3 & - & - & 2 & 2 & - \\
\hline & $(B)$ & & - & 2 & -3 & - & - & -3 & 2 & - \\
\hline$h_{S \bar{C}} 16_{i} 16_{j} \bar{C} \bar{C} S$ & & & -3 & - & - & -3 & 2 & - & - & 2 \\
\hline
\end{tabular}

The various operators up to dimension six and their contributions to the quark and lepton mass matrices are listed in the left part of Table1. Remarkably, the operator $16_{i} 16_{j} \bar{C} \bar{C} S$ contributes in equal measure to $h_{u}$ and $h_{\nu}^{\mathrm{D}}$, in contrast to the dimension-five operator $16_{i} 16_{j} \bar{C} \bar{C}$. From Table 1 , we read off the relations

$$
Y_{\nu}^{\mathrm{D}}-Y_{u}=h_{\bar{C}}^{[1]}-5 h_{\bar{C}}^{[45]}, \quad Y_{e}-Y_{d}=5 h_{S C}^{(B)} .
$$

Proton Decay. The couplings of the fermions to color-triplet Higgs fields give rise to the proton decay operators of mass-dimension five [24] with

$$
\Gamma \propto\left|\frac{C_{5}}{M_{H_{C}}}\right|^{2}, \quad C_{5}^{L}=Y_{q q} Y_{q l}, \quad C_{5}^{R}=Y_{u d} Y_{u e}
$$

where $\Gamma$ is the decay rate, $M_{H_{C}}$ is the mass of the color triplets, and the baryon and lepton number violating couplings are denoted as

$$
\left(\frac{1}{2} Y_{q q}^{i j} Q_{i} Q_{j}+Y_{u e}^{i j} u_{i}^{c} e_{j}^{c}\right) H_{C}+\left(Y_{q l}^{i j} Q_{i} L_{j}+Y_{u d}^{i j} u_{i}^{c} d_{j}^{c}\right) \bar{H}_{C} .
$$

Decay diagrams $p \rightarrow K^{+} \bar{\nu}$ via the two distinct operators $Q Q Q L$ and $u^{c} d^{c} u^{c} e^{c}$ are sketched in the adjoining figure.

The determination of the baryon and lepton number violating couplings is important for the calculation of the decay amplitude. In SU(5), the impact of higher-dimensional Yukawa operators on these couplings, relative to the mass terms, is sufficient to reduce the decay rate by several orders of magnitude and make it consistent with the experimental upper bound [10].

The dimension-five operators are generated by integrating out the heavy color-triplet Higgs fields. In addition to the standard
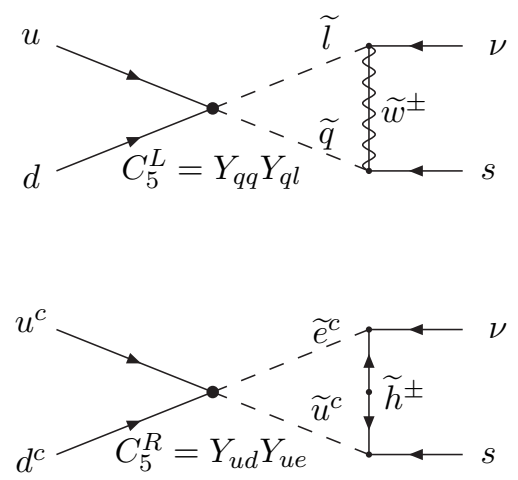
couplings to $H_{C}(T)$ and $\bar{H}_{C}(T)$, we have those to $\widetilde{H}_{C}(\bar{C})$ and $\widetilde{\bar{H}}_{C}(C)$, via the higher-dimensional operators [8]. The coefficients are listed in the right part of Table 1; note that the first two rows express the couplings to $H_{C}$ and $\bar{H}_{C}$, the remaining rows those to $\widetilde{H}_{C}$ and $\widetilde{\bar{H}}_{C}$.

Due to the two pairs of color triplets, we cannot simply read off the relations between the baryonnumber-violating couplings and the mass matrices, in contrast to $\mathrm{SU}(5)$. We find, however, relations among the four different couplings, namely

$$
Y_{q q}=Y_{u e}
$$

and the $\mathrm{SU}(5)$ relation [10, 25]

$$
Y_{u d}-Y_{q l}=Y_{d}-Y_{e}
$$

A detailed study of the various decay modes requires a numerical analysis of the fermion masses and mixings, which is beyond the scope of this letter. Note that the new decay operators due to the color triplets in $C$ and $\bar{C}$ can change the branching ratios significantly [8]. Naïve choices such as $Y_{u}=Y_{q q}$ yield a decay rate which could be in conflict with the experimental bounds [26], provided that the color triplets have GUTscale masses. A study along the lines of Ref. [10], however, would provide means to control the total decay rate. The experimental bounds on proton decay, together with the observed pattern of fermion masses and mixings will constrain the structure and size of the various couplings.

Concluding Remarks. We have shown that $\mathrm{SO}(10)$ can be broken to $\mathrm{SU}(3)_{C} \times \mathrm{U}(1)_{\mathrm{EM}}$ by a set of four Higgs fields: $S(54), C(16), \bar{C}(\overline{16})$, and $T(10)$. This raises the question of whether this scenario is the minimal set of Higgs fields capable of breaking $\mathrm{SO}(10)$ to $\mathrm{SU}(3)_{C} \times \mathrm{U}(1)_{\mathrm{EM}}$. If we replace the symmetric tensor $S(54)$ by the antisymmetric tensor, $A(45)$, will this system work as well?

In order for $A$ to acquire a vev, we need a quartic coupling, such as $\frac{1}{M} \operatorname{tr} A^{4}$. Even if it acquires a vev in the $B-L$ direction, the Dimopoulos-Wilczek form is destabilized when $A$ couples to the spinors via the operator $C A \bar{C}$ and the $I_{3 R}$ component gets a vev as well [0, 12]. (If $A$ acquires a vev in the hypercharge direction, both the $B-L$ and $I_{3 R}$ components of $A$ have a vev from the beginning.) Moreover, this coupling generates a splitting among the electroweak doublets and color triplets in $C$ and $\bar{C}$. Hence, although there is not a renormalizable coupling of $A$ to $T$, the doublet-triplet splitting emerges and fine-tuning allows to arrange for two light weak doublets, while the color triplets are heavy. Therefore this scenario is capable of breaking $\mathrm{SO}(10)$ to $\mathrm{SU}(3)_{C} \times \mathrm{U}(1)_{\mathrm{EM}}$ as well.

Comparing the two scenarios with either $A(45)$ or $S(54)$, we notice that in order to reproduce fermion masses, we only need operators of dimension five in the former case. In the latter scenario, however, we have seen that the dimension-six operators are necessary, and have a significant impact. Dimension-six operators, although not necessary, could have a significant impact in the models with $A(45)$ as well. Furthermore, looking at Table 1, we note that several operators contribute identically to the fermion mass matrices. This reduces the number of free parameters in these matrices. Hence, using $S(54)$ is a promising alternative to the already established scenarios.

We are grateful for valuable discussions with K. Babu and to C. Albright for comments on the manuscript. This work was supported in part by the U. S. Department of Energy under contract No. DE-FG0291ER40677. 


\section{References}

[1] H. Georgi, in: Particles and fields (ed. C. Carlson), AIP Conf. Proc. 23, 575 (1975);

H. Fritzsch and P. Minkowski, Annals Phys. 93 (1975) 193.

[2] H. Georgi and S. L. Glashow, Phys. Rev. D 6, 429 (1972).

[3] T. E. Clark, T. K. Kuo and N. Nakagawa, Phys. Lett. B 115, 26 (1982);

C. S. Aulakh and R. N. Mohapatra, Phys. Rev. D 28, 217 (1983);

C. S. Aulakh, B. Bajc, A. Melfo, G. Senjanovic and F. Vissani, Phys. Lett. B 588, 196 (2004);

B. Bajc, A. Melfo, G. Senjanovic and F. Vissani, Phys. Rev. D 70, 035007 (2004).

[4] A. Melfo, arXiv:0705.4059 [hep-ph] and references therein.

[5] C. S. Aulakh and S. K. Garg, arXiv:hep-ph/0612021;

W. Grimus and H. Kuhbock, arXiv:hep-ph/0612132.

[6] D. Chang, T. Fukuyama, Y. Y. Keum, T. Kikuchi and N. Okada, Phys. Rev. D 71, 095002 (2005).

[7] S. M. Barr and S. Raby, Phys. Rev. Lett. 79, 4748 (1997).

[8] K. S. Babu, J. C. Pati and F. Wilczek, Phys. Lett. B 423, 337 (1998); Nucl. Phys. B 566, 33 (2000).

[9] C. H. Albright and S. M. Barr, Phys. Rev. D 58, 013002 (1998);

C. H. Albright, K. S. Babu and S. M. Barr, Phys. Rev. Lett. 81, 1167 (1998);

C. H. Albright and S. M. Barr, Phys. Lett. B 452, 287 (1999); ibid 461, 218 (1999); Phys. Rev. Lett. 85, 244 (2000); Phys. Rev. D 62, 093008 (2000);

S. Wiesenfeldt, Phys. Rev. D 71, 075006 (2005).

[10] D. Emmanuel-Costa and S. Wiesenfeldt, Nucl. Phys. B 661, 62 (2003).

[11] S. Dimopoulos and F. Wilczek, in: The Unity of the Fundamental Interactions, Proceedings of the 19th Course of the International School of Subnuclear Physics, Erice, Italy, 1981, edited by A. Zichini (Plenum Press, New York, 1983) 237-249;

R. N. Cahn, I. Hinchliffe and L. J. Hall, Phys. Lett. B 109, 426 (1982).

[12] K. S. Babu and S. M. Barr, Phys. Rev. D 48, 5354 (1993).

[13] For $\mathrm{SO}(10)$ orbifold GUT models and their phenomenology, see, e.g.:

T. Asaka, W. Buchmüller and L. Covi, Phys. Lett. B 523, 199 (2001);

L. J. Hall, Y. Nomura, T. Okui and D. R. Smith, Phys. Rev. D 65, 035008 (2002);

R. Dermisek and A. Mafi, Phys. Rev. D 65, 055002 (2002);

W. Buchmüller, L. Covi, D. Emmanuel-Costa and S. Wiesenfeldt, JHEP 0409, 004 (2004).

[14] R. N. Mohapatra, Unification and Supersymmetry (Springer, New York, 3rd ed., 2003);

P. Binetruy, Supersymmetry: Theory, Experiment and Cosmology (Oxford University Press, Oxford, 2006).

[15] G. Lazarides, M. Magg and Q. Shafi, Phys. Lett. B 97, 87 (1980);

G. Lazarides, Q. Shafi and C. Wetterich, Nucl. Phys. B 181, 287 (1981);

F. Buccella, L. Cocco and C. Wetterich, Nucl. Phys. B 243, 273 (1984).

[16] Z. y. Zhao, J. Phys. G 8, 1019 (1982).

[17] See, e.g., K. S. Babu and E. Ma, Phys. Rev. D 31, 2316 (1985);

K. S. Babu and S. M. Barr, Phys. Rev. D 50, 3529 (1994);

C. S. Aulakh, B. Bajc, A. Melfo, A. Rasin and G. Senjanovic, Nucl. Phys. B 597, 89 (2001).

[18] K. S. Babu, I. Gogoladze, P. Nath and R. M. Syed, Phys. Rev. D 72, 095011 (2005).

[19] K. S. Babu, I. Gogoladze, P. Nath and R. M. Syed, Phys. Rev. D 74, 075004 (2006).

[20] V. Barger, J. Jiang, P. Langacker and T. Li, arXiv:hep-ph/0612206.

[21] J. Sayre and S. Wiesenfeldt, Phys. Lett. B 637, 295 (2006).

[22] K. S. Babu and S. M. Barr, Phys. Rev. Lett. 85, 1170 (2000);

[23] For more details, see, e.g. G. G. Ross, Grand Unified Theories (Benjamin/Cummings, 1984).

[24] N. Sakai and T. Yanagida, Nucl. Phys. B 197, 533 (1982);

S. Weinberg, Phys. Rev. D 26, 287 (1982).

[25] B. Bajc, P. Fileviez Perez and G. Senjanovic, arXiv:hep-ph/0210374.

[26] K. Kobayashi et al. [Super-Kamiokande Collaboration], Phys. Rev. D 72, 052007 (2005). 